\title{
Research in Fracture Healing and Its Clinical Applications in the Veterinary Practice
}

\author{
Mafamane $\mathrm{H}^{1}$, Hackenbroich $\mathrm{C}^{1}$, Ellinghaus $\mathrm{A}^{\star 2}$ and Schmidt-Bleek $\mathrm{K}^{2,3}$ \\ ${ }^{1}$ Animal Clinic Northeim, Northeim, Germany \\ ${ }^{2}$ Julius Wolff Institut and Center for Musculoskeletal Surgery, Charité - Universitätsmedizin Berlin, Germany \\ ${ }^{3}$ Berlin-Brandenburg Center for Regenerative Therapies, Charité - Universitätsmedizin Berlin, Germany
}

${ }^{*}$ Corresponding author: Ellinghaus A, Julius Wolff Institut, Charité - Universitätsmedizin Berlin, Augustenburger Platz 1, 13353 Berlin, Germany, Tel: +49 3045065 9027, E-mail: agnes.ellinghaus@charite.de

Citation: Mafamane H, Hackenbroich C, Ellinghaus A, Schmidt-Bleek K (2017) Research in Fracture Healing and Its Clinical Applications in the Veterinary Practice. J Vet Sci Ani Husb 5(3): 303

Received Date: July 14, 2017 Accepted Date: October 24, 2017 Published Date: October 26, 2017

\begin{abstract}
Bone healing is a complex process consisting of multiple sequential phases needing the coordinated interaction of various cells, growth factors, cytokines and mechanical conditions. However, bone healing is one of the rare regenerative processes resulting in complete restoration of form and function. Fracture treatment aims towards a swift return to functional use of the injured limb. Therefore stabilization is a key requisite. In the veterinary clinic difficult fracture cases often require a unique stabilization by custom made external fixation. While this fixation technique harbors risk factors it offers a high flexibility essentially needed to successfully treat small animal fracture patients. Here we draw the connection between the up-to-date knowledge in bone healing and case reports of external fixation techniques from the small animal practice with respect to benefits and risk factors.
\end{abstract}

Keywords: Fracture; External Fixation; Mechanical Stability; Custom Made Fixation

\section{Introduction}

Fracture healing is a regenerative process in which bone has the unique capacity of healing without scar tissue formation which results in unscarred restoration of tissue integrity [1]. Sequential and overlapping stages of regeneration can be distinguished in the process of healing [2] beginning with a hematoma/ inflammation phase, transitioning through a callus phase and woven bone formation before remodelling restores the bone to pre-injury conditions. This description of the fracture healing process however is highly simplified. The inflammatory phase for example begins with a pro-inflammatory signalling cascade that is essential for the initiation of the healing process. This pro-inflammatory reaction is phylogenetically coupled to the coagulation pathway during hematoma formation [3]. Consecutively the downregulation of the inflammatory reaction is important to allow the essential revascularisation process to take place to restore the supply towards the injury site [4]. This interdependency of the inflammatory and angiogenic process is tightly regulated during successful bone healing and thus also prone for failure [5]. The hypoxia that results from the disruption of the blood vessels during the injury is another important factor that regulates the angiogenic process in the early fracture healing. The transcription factor HIF1a (hypoxia induced transcription factor 1 alpha) that is upregulated due to the hypoxic conditions in the early bone healing phase induces the expression of VEGF (vascular endothelial growth factor) that is essential for the revascularization process [6,7]. The unfavourable conditions in the early phase of bone healing with hypoxia, low $\mathrm{pH}$, high potassium and sodium concentration are difficult for specific cell subsets; however macrophages adapt and function during this phase as well as T cells, also demonstrating the tight interconnectivity of the immune reaction and the revascularisation step necessary for successful healing [8,9]. The complexity of the healing process continues until the bone is remodelled. Sequential and overlapping phases constitute the process where a multitude of cells (mesenchymal stromal cells, immune cells of the innate and adaptive immune system, osteoblasts, osteocytes, osteoclasts, fibroblasts, fibrocytes, chondrocytes, progenitor cells, blood cells) and their signalling molecules (cytokines, chemokines, growth factors) interact to re-establish the integrity of the cancellous/ cortical bone with the periosteum, endosteum, bone marrow cavity and the vasculature and nerve structure to rebuilt bone. At the very beginning of the healing process the bone is being treated and repositioned to allow an optimal healing outcome.

Treating fractures with a stabilization of the bone can be retraced at least 2400 years before Christ, proven by excavations in Egypt [10]. Therefore, immobilization seems to be the most important aspect to enable bone healing. Without stabilization, for example in the wild, bone fracture and especially long bone fracture often results in the demise of the afflicted individual because without 
the load bearing ability of one of the extremities survival becomes challenging. Surprisingly, younger animals seem to have a slight chance of survival [11]. In these cases, however, the bone often heals with a severe misalignment due to the missing immobilization and stabilization. Fracture treatment has developed from the simple splinting, which today would equal a cast stabilization, towards more complex internal fixation techniques with plates or nails with procedures such as ORIF (open repositioning and internal fixation), CRIF (closed repositioning and internal fixation), or the MIPO (minimal invasive plate osteosynthesis). Another technique used in fracture fixation is the external fixator with its most prominent example of the Ilizarov frame. Prof. Gavriil Abramovich Ilizarov treated soldiers returning with fractures sustained during world war II and successfully used this fixation technique on a tibial non-union in 1954 [12]. The unilateral external fixation has a longer history, being first mentioned in 1840 by Jean-Francois Malgaigne who realized fixation of a fractured tibia with spikes driven into the bone an external strap fixation [13].

Fracture stabilization in the veterinary practice faces the difficulty of the varying size of the patients. Especially in dogs the different races from Chihuahua to Great Danes present high demands on the flexibility of the fixation systems [14]. This is always coupled with the fact that the treatment has to be affordable for the pet owner for whom the veterinary bill is often a severe challenge [14]. Therefore, fixation systems used for fracture treatment in the human medical clinic are often not available for the veterinarian.

Fracture treatment should aim to stabilize the bone, reestablish anatomical structures and allow immediate mobilization while allowing bony consolidation. The aim of direct healing through exact anatomical repositioning without fracture gap and exclusion of intra-fragmentary movement is not always possible, especially in complex fractures. Therefore, most fractures heal through indirect or secondary healing [15]. Recent development in fracture treatment aims to conserve the original fracture hematoma, for example the MIPO technique fulfills this strategy [6].

An introduction in the bone healing biology will be given using results from animal experimental studies. This will be compared to bone healing after external fixation in the veterinary practice using case reports to highlight the beneficial aspects but also considering the risks.

\section{Materials and Methods}

\section{Fracture Healing Process in Mice}

Fracture healing was monitored in mice at day 3, 7, 14, 21, and 28. In C57/BL6, aged 8-10 weeks, male mice ( $\mathrm{n}=8$ ) a standardized unilateral closed fracture was performed in the left femur. Animals were anesthetized with isoflurane/oxygen and received pain medication (Buprenorphin 1mg/kg BW i.p., Reckitt Benckiser, Mannheim, Germany). After skin incision the patella was dislocated to the lateral side and a passage for an intramedullary pin was created using a hollow needle (Microlance 3, $0.55 \mathrm{x} 25 \mathrm{~mm}, \mathrm{BD}$ Drogheda, Ireland). An intramedullary pin (Thermo spinal needle 17, 0.5 x 0.9 mm, TERUMO EUROPE N. V., Leuven, Belgium) was inserted into the bone marrow cavity and a three point bending fracture was induced according to Bonnarens and Einhorn [16]. All animal experiments were carried out according to the policies and principles established by the Animal Welfare Act, the $\mathrm{NIH}$ Guide for Care and Use of Animals and the national animal welfare guidelines. The study was approved by the local legal representative (Landesamt für Gesundheit und Soziales, Berlin: G0206/08).

\section{Histological Analyses}

Femurs were harvested at the designated time points for histological analyses and fixed in $4 \%$ paraformaldehyde for 48 hours at $4{ }^{\circ} \mathrm{C}$. Decalcification of the bones was performed using a 1:2 solution of $4 \%$ PFA and $14 \%$ EDTA at $4{ }^{\circ} \mathrm{C}$ for 3 weeks. The solution was changed every 3 days. After dehydration bones were embedded in paraffin $(n=8)$ and cut into $4 \mu$ m thick slices. Sections were stained with Movat pentachrome [17], which allows a distinct and colorful contrast between the different tissue types: hematoma/ fibrin is stained different shades of red, cartilage is deep green, fibrous connective tissue is light green-blue and bony tissue is stained yellow.

\section{Fixation Stiffness}

For unstable fixation the intramedullary pin was used as described above. For stable fixation of the femur the MouseExFix (RISystem, Davos, Switzerland) was used in C57/BL6, aged 8-10 weeks, female mice $(n=6)$ under the same anaesthesia regime mentioned above. A lateral longitudinal incision of the skin $(2 \mathrm{~mm})$ from knee to hip was performed for a mid-diaphyseal approach to the femur. The femur was exposed by blunt dissection of Musculus vastus lateralis and Muscculus biceps femoris. Four pins were placed $(0.45 \mathrm{~mm}$ diameter) through the connector bar of the external fixator (MouseExFix, RISystem, Davos, Switzerland) positioning the external fixator antero-laterally in parallel to the femur. A $0.70 \mathrm{~mm}$ osteotomy was performed between the medial pins using a Gigli wire saw (RISystem, Davos, Switzerland). Postoperative analgesia was conducted with tramadol hydrochloride added to the drinking water $(25 \mathrm{mg} / \mathrm{L})$. The study was approved by the local legal representative (Landesamt für Gesundheit und Soziales, Berlin: G0008/12). After 21 days of healing, bones were analysed using microcomputer tomography to document mineral bone formation. Bones were scanned with a fixed isotropic voxel size of $10.5 \mu \mathrm{m}$ (Viva40 micro-CT, Scanco Medical AG ${ }^{\oplus}$, Switzerland, $70 \mathrm{KVp}, 114 \mu \mathrm{A}$ ). A fixed global threshold of $190 \mathrm{mg} \mathrm{HA} / \mathrm{cm}^{3}$ (9 week old male mice) and $240 \mathrm{mg} \mathrm{HA} / \mathrm{cm}^{3}$ (12 week old female mice) was selected that allowed the rendering of mineralized callus only. For the larger callus with unstable fixation a minimum of 630 slices $\left(630^{\star} 10.5 \mu \mathrm{m}\right)$ was chosen such that the fracture callus was completely included. With stable fixation the VOI included 
190 slices. All analyses were performed on the digitally extracted callus tissue using 3D distance techniques (Scanco ${ }^{\circledR}$ software, Switzerland) [18].

\section{Sheep Study to Investigate Periost Reaction upon Bone Injury}

Skeletally mature female sheep ( $n=6 /$ per time point) underwent an osteotomy stabilized with an external fixator [19]. A customized unilateral external fixator was mounted on the unfractured tibia under general anesthesia. Subsequently a $2 \mathrm{~cm}$ bone fragment was removed mid diaphyseally. Sheep were provided with potent analgesics i.v. (Fentanyl, Jansen ${ }^{\oplus}$-Cilag GmbH, Neuss, Germany) intra-operatively, after surgery the animals were provided with Fentanyl plasters (50 $\mathrm{g} / \mathrm{h}$, Jansen -Cilag GmbH, Neuss, Germany) for the time until sacrifice after 12 hours by an overdose of thiopental-sodium (Trapanal ${ }^{\circledR} 2.5 \mathrm{~g}$, Altana Pharma GmbH, Konstanz, Germany) and $100 \mathrm{ml}$ potassium-chloride (1M Kaliumchlorid-Lösung Fresenius, Bernburg, Germany). All animal experiments were carried out according to the policies and principles established by the Animal Welfare Act, the NIH Guide for Care and Use of Animals and the national animal welfare guidelines. The study was approved by the local legal representative (Landesamt für Gesundheit und Soziales, Berlin: G00332/05, G0127/07). Periost was harvested directly adjacent to the osteotomy side and from an unfractured control bone. For histological analysis, the samples were embedded in paraffin (after PFA fixation) and $2 \mu \mathrm{m}$ thick sections were cut. Sections were stained with hematoxylin-eosin.

\section{Results}

\section{Fracture Healing is a Multistage Process}

Each injury induces an endogenous repair program with the ultimate goal of restoration of the disrupted tissue continuum. In many tissues the regeneration of the original tissue is not possible and a replacement in the form of a scar tissue evolves. This is often coupled with a reduced function. However, in a few tissues in mammals the regenerative capacity still exists and bone, in most cases, is able to regenerate its form and function. This regenerative process is a multistage process (Figure 1). Healing starts with hematoma formation that is coupled with an inflammatory reaction, the hematoma matures towards granulation tissue from which a soft callus generates that is dominated by cartilage, cartilage becomes hypertrophic and woven bone formation is initiated when the callus transforms towards a hard callus. After bony bridging of the defect the remodeling starts that aims at restoring form and function of the fractured bone according to the mechanical cues which are sensed by the bone upon movement.

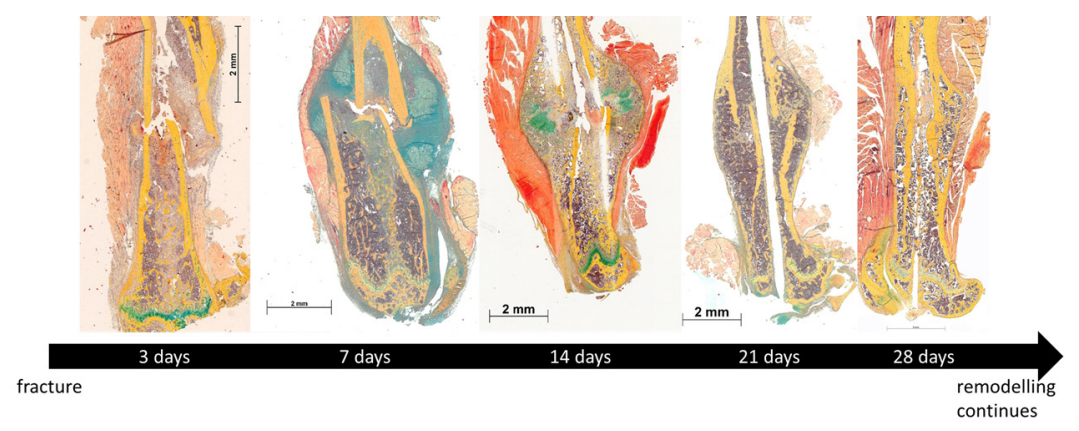

As representatives for the fracture healing phases 5 time points of the healing cascade are shown: 3 days: hematoma and inflammatory phase; 7 days: end of inflammatory phase - beginning of cartilage (hyaline) phase; 14 days: soft callus phase with cartilage ends hypertrophic cartilage with beginning of woven bone formation; 21 days: fracture is consolidated, woven bone throughout the fracture region. No cartilage remains; 28 days: remodeling phase. Histological samples stained with Movat Pentachrome: bone = yellow; bone marrow $=$ purple; cartilage $=$ blue-green, muscle $=$ orange; connective tissue $=$ light blue $[2,4,6,15,34-43]$.

Figure 1: Fracture healing process in a mouse 3 point bending fracture model of the femur

\section{Bone Forming Processes}

Bone formation during fracture healing recapitulates developmental processes [20]. Embryological, the limb skeleton generates

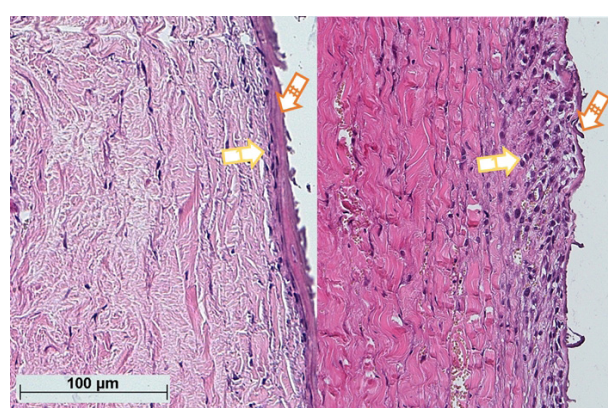

Periost has been carefully elevated from the bone and was stained with hematoxylin eosin. Left: periost in an uninjured bone; right: periost adjacent to a bone osteotomy 60 hours after injury induction (sheep). Orange arrow (\#): remnants of the bone indicating that the periosteum remained intact during the harvesting procedure, Yellow arrow (1): indicating the width of the periosteum. Activation of the periosteum upon bony injury is clearly visible in the increased cellular density in the right image.

Figure 2: Bone injuries cause a change in the cellular structure of the Periost 
from the lateral plate mesoderm while the neural crest gives rise to the skull. The craniofacial bones are formed by intramembranous ossification, a process that also forms the basis of the width growth of long bones. Long bone grows wider due to cells on the inner surface of the periosteum (a membrane of compact mesenchymal cells covering the bone surface) which differentiate in to osteoblasts and directly form new bone by intramembranous ossification. To this respect the periosteum plays a very active part during bone healing. The activation upon injury is apparent in the high cellularity of the periosteum adjacent to an osteotomy side (Figure 2).

The embryonic process of the limb bone development proceeds through differentiation of mesenchymal cells into cartilage which is later replaced by bone [20]. This cartilaginous intermediate is also seen in endochondral bone formation. This form of bone healing occurs in fractured bone in cases where an anatomical repositioning and stable fixation of the fractured bone ends is not possible so that either intrafragmentary movement or a gap remain. Bone healing in these cases combines both the intramembranous ossification (Figure 3A) and the endochondral ossification process (Figure 3B) in order to successfully heal the fracture [2].

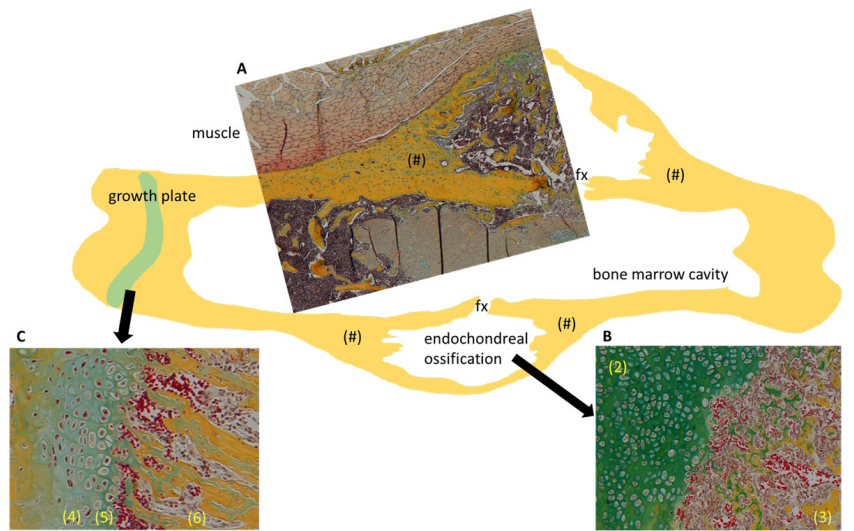

In secondary fracture healing two bone formation processes occur in parallel. The periosteum initiates intramembranous bone formation without a cartilaginous intermediate (A: (\#) intramembranous bone formation originating from the periosteal reaction) while gap bridging requires cartilage formation, maturation and consecutive woven bone formation (B: (2) cartilage, (3) woven bone). This endochondral bone formation process also occurs during length growth of long bones in the growth plate (C: (4) column cartilage, (5) hypertrophic cartilage, (6) newly formed bone trabeculae). The contours of the mouse femur from which these images originate are sketched in yellow, (\#) indicates intramembranous ossification, $\mathrm{fx}$ indicates the fracture site. Histological images are stained with Movat Pentacrome (bone= yellow, bone marrow $=$ purple, cartilage $=$ blue-green, erythrocytes $=$ bright red) .

Figure 3: Intramembranous versus endochondral ossification

Distal tibia fractures in small animals (dogs and cats) often successfully heal through secondary bone formation as shown in a clinical case report of a cat (3kg, 1 year) (Figure 4). Stabilization was achieved with a unilateral external fixator with three pins distally and 3 pins proximally connected with a technovit bar [21]. The fracture area was left undisturbed, the original hematoma remained and the bone fragments and a gap between the main fractures ends were not corrected [6]. Healing was achieved in 7 weeks and proceeded through a considerable visible mineralized callus as indicated in Figure 4.
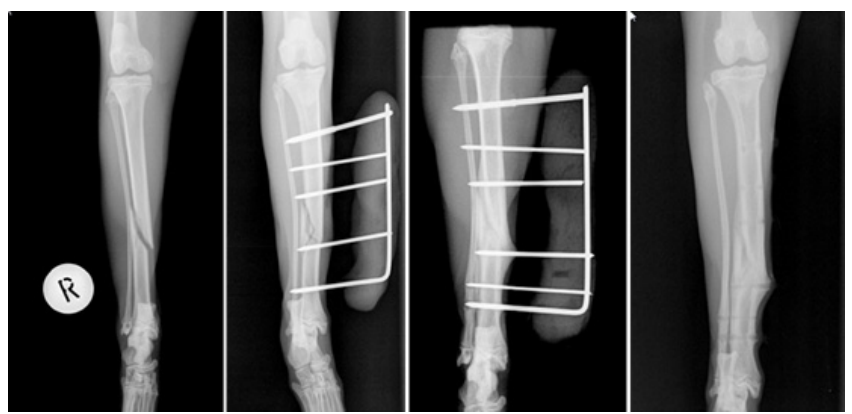

Fracture healing in a 1 year old cat under external fixation. From left to right: Tibia fracture 14.06.2012; external fixation 15.06.2012; healing after 7 weeks 02.08.2012, tibia after removal of external fixation 02.08.2012.

Figure 4: X-ray scan of the cat right fractured tibia stabilized with external fixation

\section{Discussion}

\section{Fixation - Which Stability to Favor?}

The fixation used to stabilize a fractured bone is the most important tool in the clinic to facilitate the healing process and allow early mechanical loading of the injured limb [15]. In most cases the fixation is determined by the bone which is fractured and by the type of fracture (e.g. transverse fracture, oblique fracture, comminuted fracture, impacted fracture ...). While fixation techniques are highly standardized in the human clinic, in veterinary practice sometimes unconventional fixation has to be applied due to the highly diverse clientele (Figure 5). 

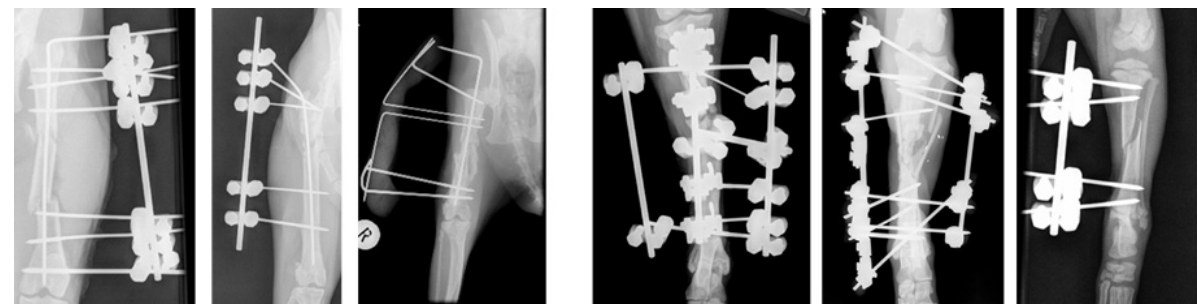

Examples of external fixation techniques from the veterinary practice: left: external fixation of femur fractures in cats $(3 \mathrm{x})$, right: external fixation of tibia fractures in dogs $(3 \mathrm{x})$.

Figure 5: Custom made external fixation systems

The fixation stability determines the fracture callus formation [22]. With an unstable fixation the callus formation is enhanced, while higher fixation stiffness reduces the callus circumference (Figure 6). This is due to the fact that cartilage as a substitute for the slower formed mineralized tissue has a lower stiffness and thus compensated for this lack by enlarging the diameter to quickly regain load bearing.
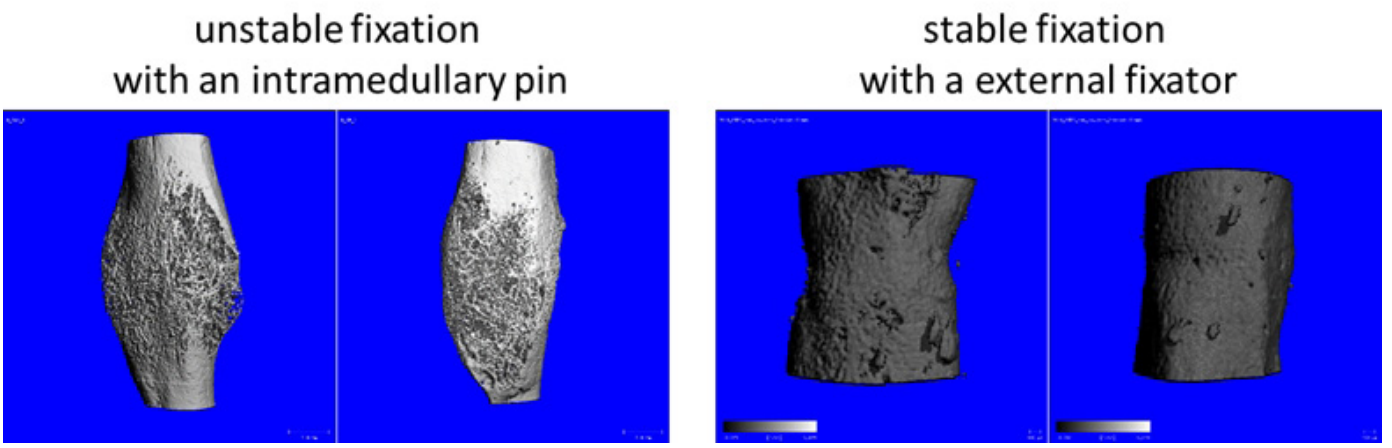

Fixation stability highly impacts the healing course. The size of the callus formed to stabilize the injured bone is dependent on the fixation stability: An unstable fixation with an intramedullary pin of a small diameter leads to a large callus formation 21 days after 3 point bending fracture of the femur in a mouse fracture healing model. Shown are two different animals as representative examples of unstable fixation (left). A stable fixation with an external fixator (RISystem, MouseFixEx, Davos, Switzerland) shows no diameter enlargement in an $0.7 \mathrm{~mm}$ osteotomy model of a mouse femur after 21 days of healing. Shown on the right side are two representative examples. Scale bar left $500 \mu \mathrm{m}$, right $100 \mu \mathrm{m}$

Figure 6: Influence of fixation stability

The question arises whether the immediate use of the treated injured bone could be beneficial for the healing process. Recently the interdependency of the BMP (bone morphogenetic protein) pathway and mechanical stimuli has been investigated [23]. BMP signaling is enhanced under mechanical stimulation. As BMP is a very potent inducer of bone formation this supports the idea of an early mobilization in fracture patients which is almost always the case in the veterinary practice. A mechanical stimulation has also recently been investigated using a massage-like compression to enhance muscular healing with positive results [24]. The idea of a change in fixation stability, even though investigated for some time now in a research setting has so far not reached the clinic [25-28]. More often the change in the fixation regime is dictated by an unsatisfactory healing progression [29]. While a plate fixation is preferable to an external fixation it often lacks the fixation stability needed to reach a satisfactory healing result (Figure 7).
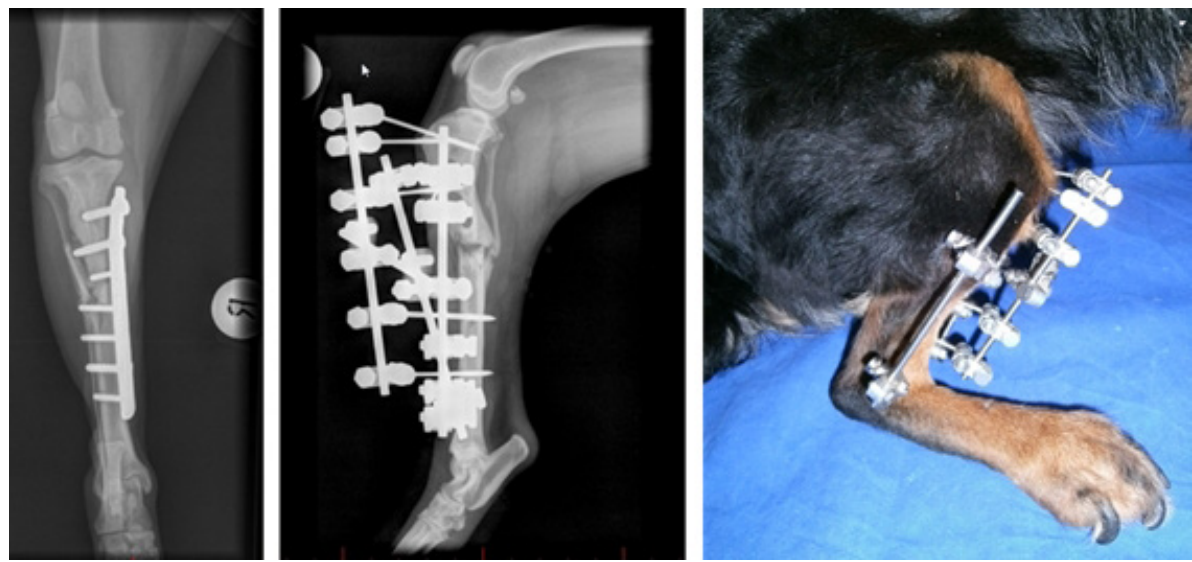

A combined fracture of tibia and fibula was primarily stabilized with a plate (left, 07.11.2013). Middle: 4 weeks later the healing progression was not satisfactory so that the fixation was changed towards an external fixator (06.12.2013 - image from 13.03.2014) - the external fixation was removed after about 9 month (22.08.2014). Right: The external fixation needs to be light, easy to clean, durable and should retain the use of the limb.

Figure 7: Case report 2: dog with tibia and fibula fracture 


\section{External Fixation Bears Risks}

A risk factor especially associated with external fixation is an infection [30,31]. Due to the pin fixation the protective shell of the skin is disrupted and the pin channels offer an entry point for pathogens. Pin channel infections have been reported to occur in more than 50\% of cases [32]. A thorough treatment with antibiotics and antimicrobial solutions resolves this side effect in most cases. A case report: a young dog with an open fracture of the femur (first grade). Due to the impact the femur showed multiple lengthwise cracks, a soft tissue swelling and a disruption of the V. saphena were present (Figure 8). Two unilateral external fixators were mounted on the femur. Subsequently the fracture area was highly infected. Anti-infective treatment was successfully applied and bony consolidation was reached within 4 weeks. In this case however the healed bone showed a shortening of the limb in the end.
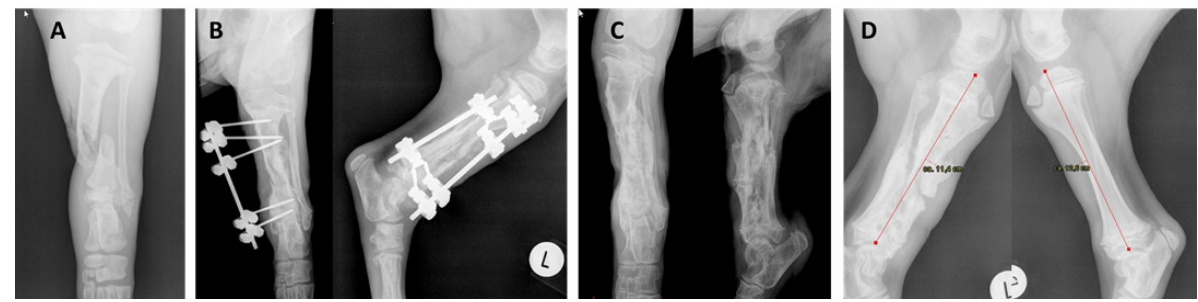

A 9 week old german shepard with an open tibia fracture. A) Fracture 11.12.2013; B) stabilized with an external fixator (left 27.12.2013, right 19.12.2013); C) bone after removal of the fixator (03.01.2014); D) limb length measurement, left tibia 11,4cm and right healthy tibia $12,6 \mathrm{~cm}$. Due to the young age of the patient the fixator had to be removed after only 3 weeks. Even though the bone was not consolidated by that time and the tibia was visibly shorter compared to the other side the bone recovered to full functionality a short time later.

Figure 8: Case report 3: tibia fracture in a puppy

\section{External Fixation in Complicated Cases}

Even though the external fixation bears a high risk of infection due to the pins, it is often the method of choice in treating fractured bone in small animals [14,33]. A case often seen in the veterinary practice is the cat that fell out of an open window (Figure 9). In this case a severe comminuted fracture of the femur was successfully treated with an external fixator. With a lateral approach the soft tissue appeared severely damaged and while the distal and proximal fragments were fixated the multiple diaphyseal fragments where not disturbed to prevent further disruption of the vasculature. Due to the short distal fragments a locking nail was discarded as a treatment possibility and a unilateral external fixation was chosen. Roughly six months later the fixation was removed due to pin lysis, even though the bony consolidation was still considered weak [29]. However, in this case the femur consumed full weight bearing after implant removal and the cat recovered.
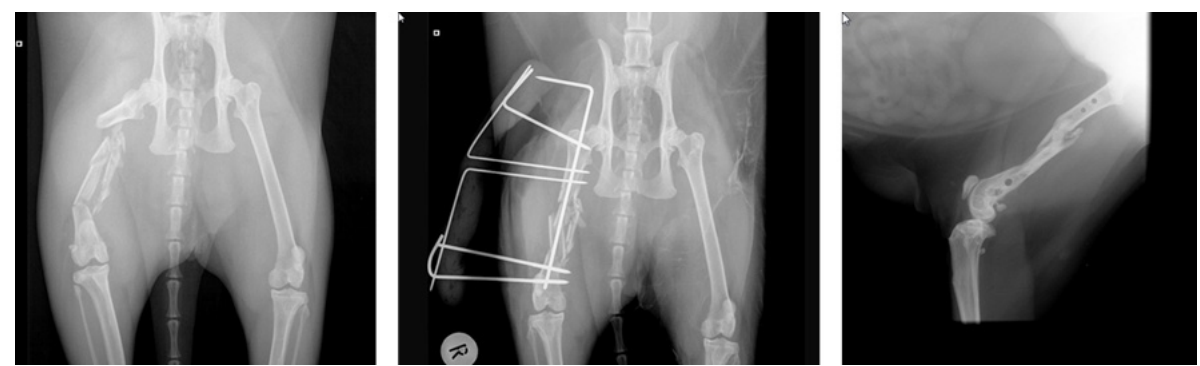

A comminuted femoral fracture in a cat was stabilized with an external fixator. Fixation was removed after 9 months due to increasing pin lysis. By this time consolidation was achieved and the animal recovered.

Figure 9: Case report 4: cat with femur fracture

Fractures that occur in the proximity of joints represent a difficult situation with respect to the fixation. However, also for these cases the flexible external fixation system of the veterinary praxis leads to successful healing. The cat Emil had to be treated twice and recovered (Figure 10).
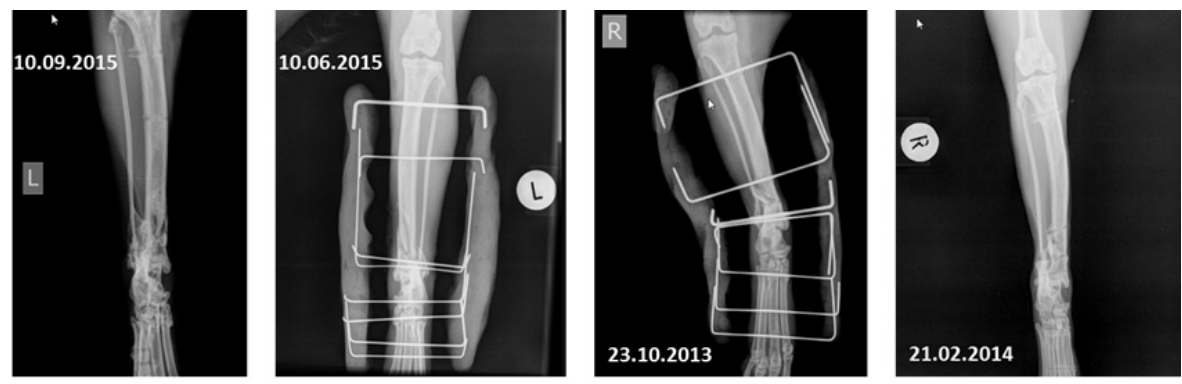

The cat Emil suffered from a distal fracture of the tibia not once but twice and was successfully treated both times with an external fixator.

Figure 10: Case report 5: cat with tibia fracture 


\section{Conclusion}

Fracture healing represents a highly complex process depending on multiple factors to achieve a satisfactory outcome. However, the high regenerative potential of bone enables the reestablishing of load bearing even in complicated cases when stabilization can be achieved. And even though it sometimes takes considerable time this process can overcome complications that are common in the veterinary practice. Among these complicated fracture situations with comorbidities like swelling, soft tissue trauma and disrupted vascularization, infection and pin loosening. When stabilized with a fixation system that withstands the lacking compliance of the patient that wants to resume load bearing quickly a successful healing is possible and probable.

\section{Acknowledgements}

This study was supported by a grant from the German Research Foundation (DFG SCHM 2977) and by the Berlin-Brandenburg Center for Regenerative Therapies (BCRT). We would like to acknowledge Norma Schulz for her excellent technical support.

\section{References}

1. Einhorn TA (1998) The cell and molecular biology of fracture healing. Clin Orthop Relat Res 355: S7-21.

2. Schmidt-Bleek K, Petersen A, Dienelt A, Schwarz C, Duda GN (2014) Initiation and early control of tissue regeneration - bone healing as a model system for tissue regeneration. Expert Opin Biol Ther 14: 247-59.

3. Opal SM (2000) Phylogenetic and functional relationships between coagulation and the innate immune response. Crit Care Med 28: S77-80.

4. Schmidt-Bleek K, Schell H, Schulz N, Hoff P, Perka C, et al. (2011) Inflammatory phase of bone healing initiates the regenerative healing cascade. Cell Tissue Res 347: 567-73.

5. Schmidt-Bleek K, Kwee BJ, Mooney DJ, Duda GN (2015) Boon and Bane of Inflammation in Bone Tissue Regeneration and Its Link with Angiogenesis. Tissue Eng Part B Rev 21: 354-64.

6. Schell H, Duda GN, Peters A, Tsitsilonis S, Johnson KA, et al. (2017) The haematoma and its role in bone healing. J Exp Orthop 4: 5.

7. Gaber T, Dziurla R, Tripmacher R, Burmester GR, Buttgereit F (2005) Hypoxia inducible factor (HIF) in rheumatology: low O2! See what HIF can do! Ann Rheum Dis 64: 971-80.

8. Gaber T, Schellmann S, Erekul KB, Fangradt M, Tykwinska K, et al. (2011) Macrophage migration inhibitory factor counterregulates dexamethasone-mediated suppression of hypoxia-inducible factor-1 alpha function and differentially influences human CD4+ T cell proliferation under hypoxia. J Immunol 186: 764-74.

9. Street J, Winter D, Wang JH, Wakai A, McGuinness A, et al. (2000) Is human fracture hematoma inherently angiogenic? Clin Orthop Relat Res 378: 224-37.

10. Smith GE (1908) The most ancient splints. Br Med J 1: 732-6.

11. Bulstrode C, King J, Roper B (1986) What happens to wild animals with broken bones? Lancet 1: 29-31.

12. Spiegelberg B, Parratt T, Dheerendra SK, Khan WS, Jennings R, et al. (2010) Ilizarov principles of deformity correction. Ann R Coll Surg Engl 92: 101-5.

13. Vidal J (1983) External fixation. Yesterday, today, and tomorrow. Clin Orthop Relat Res 180: 7-14.

14. Beale B (2004) Orthopedic clinical techniques femur fracture repair. Clin Tech Small Anim Pract 19: 134-50.

15. Bucher CH, Lei H, Duda GN, Volk H-D, Schmidt-Bleek K (2016) The Role of Immune Reactivity in Bone Regeneration. Advanced Techniques in Bone Regeneration 10.5772/62476.

16. Bonnarens F, Einhorn TA (1984) Production of a standard closed fracture in laboratory animal bone. J Orthop Res 2: 97-101.

17. Movat HZ (1955) Demonstration of all connective tissue elements in a single section; pentachrome stains. AMA Arch Pathol 60: 289-95.

18. Hildebrand T, Rüegsegger P (1997) Quantification of Bone Microarchitecture with the Structure Model Index. Comput Methods Biomech Biomed Engin 1: $15-23$.

19. Schell H, Thompson MS, Bail HJ, Hoffmann JE, Schill A, et al. (2008) Mechanical induction of critically delayed bone healing in sheep: radiological and biomechanical results. J Biomech 41: 3066-72.

20. Runyan CM, Gabrick KS (2017) Biology of Bone Formation, Fracture Healing, and Distraction Osteogenesis. J Craniofac Surg 28: 1380-9.

21. Martinez SA, Arnoczky SP, Flo GL, Brinker WO (1997) Dissipation of heat during polymerization of acrylics used for external skeletal fixator connecting bars. Vet Surg: 290-24.

22. Willie B, Adkins K, Zheng X, Simon U, Claes L (2009) Mechanical characterization of external fixator stiffness for a rat femoral fracture model. J Orthop Res 27: 687-93.

23. Kopf J, Petersen A, Duda GN, Knaus P (2012) BMP2 and mechanical loading cooperatively regulate immediate early signalling events in the BMP pathway. BMC Biol 10: 37.

24. Cezar CA, Roche ET, Vandenburgh HH, Duda GN, Walsh CJ, et al. (2016) Biologic-free mechanically induced muscle regeneration. Proc Natl Acad Sci U S A 113: 1534-9.

25. Glatt V, Evans CH, Tetsworth K (2016) A Concert between Biology and Biomechanics: The Influence of the Mechanical Environment on Bone Healing. Front Physiol 7: 678 .

26. Willie BM, Blakytny R, Glöckelmann M, Ignatius A, Claes L (2011) Temporal variation in fixation stiffness affects healing by differential cartilage formation in a rat osteotomy model. Clin Orthop Relat Res 469: 3094-101.

27. Claes L, Blakytny R, Besse J, Bausewein C, Ignatius A, et al. (2011) Late dynamization by reduced fixation stiffness enhances fracture healing in a rat femoral osteotomy model. J Orthop Trauma 25: 169-74.

28. Claes L, Blakytny R, Göckelmann M, Schoen M, Ignatius A, et al. (2009) Early dynamization by reduced fixation stiffness does not improve fracture healing in a rat femoral osteotomy model. J Orthop Res 27: 22-7.

29. Jackson LC, Pacchiana PD (2004) Common complications of fracture repair. Clin Tech Small Anim Pract 19: 168-79.

30. Silverman S, Ackerman N (1978) Postoperative osteomyelitis. Mod Vet Pract 59: 843-8. 
31. Johnson KA (1994) Osteomyelitis in dogs and cats. J Am Vet Med Assoc 204: 1882-7.

32. Fragomen AT, Miller AO, Brause BD, Goldman V, Rozbruch SR (2017) Prophylactic Postoperative Antibiotics May Not Reduce Pin Site Infections After External Fixation. HSS J 13: 165-70.

33. Canapp SO (2004) External fracture fixation. Clinical techniques in small animal practice 19: 10.1053/j.ctsap.2004.09.003.

34. Lienau J, Schmidt-Bleek K, Peters A, Haschke F, Duda GN, et al. (2009) Differential regulation of blood vessel formation between standard and delayed bone healing. J Orthop Res 27: 1133-40.

35. Schmidt-Bleek K, Schell H, Kolar P, Pfaff M, Perka C, et al. (2009) Cellular composition of the initial fracture hematoma compared to a muscle hematoma: a study in sheep. J Orthop Res 27: 1147-51.

36. Kolar P, Schmidt-Bleek K, Schell H, Gaber T, Toben D, et al. (2010) The early fracture hematoma and its potential role in fracture healing. Tissue Eng Part B Rev 16: 427-34.

37. Schmidt-Bleek K, Schell H, Lienau J, Schulz N, Hoff P, et al. (2012) Initial immune reaction and angiogenesis in bone healing. J Tissue Eng Regen Med 8: 120-30.

38. Mehta M, Schmidt-Bleek K, Duda GN, Mooney DJ (2012) Biomaterial delivery of morphogens to mimic the natural healing cascade in bone. Adv Drug Deliv Rev 64: 1257-76.

39. Könnecke I, Serra A, El Khassawna T, Schlundt C, Schell H, et al. (2014) T and B cells participate in bone repair by infiltrating the fracture callus in a two-wave fashion. Bone 64: 155-65.

40. Reinke S, Geissler S, Taylor WR, Schmidt-Bleek K, Juelke K, et al. (2013) Terminally Differentiated CD8+ T Cells Negatively Affect Bone Regeneration in Humans. Sci Transl Med 5: 10.1126/scitranslmed.3004754.

41. Schlundt C, Schell H, Goodman SB, Vunjak-Novakovic G, Duda GN, et al. (2015) Immune modulation as a therapeutic strategy in bone regeneration. J Exp Orthop 2: 1 .

42. Hoff P, Gaber T, Strehl C, Jakstadt M, Hoff H, et al. (2017) A Pronounced Inflammatory Activity Characterizes the Early Fracture Healing Phase in Immunologically Restricted Patients. Int J Mol Sci 18: 10.3390/ijms18030583.

43. El Khassawna T, Serra A, Bucher CH, Petersen A, Schlundt C, et al. (2017) T Lymphocytes Influence the Mineralization Process of Bone. Front Immunol 8: 10.3389/fimmu.2017.00562. 